\title{
Dermatomal Scratching After Intramedullary Quisqualate Injection: Correlation With Cutaneous Denervation
}

\section{Citation}

Brewer, Kori L., Jeung Woon Lee, Heather Downs, Anne Louise Oaklander, and Robert P. Yezierski. 2008. “Dermatomal Scratching After Intramedullary Quisqualate Injection: Correlation With Cutaneous Denervation." The Journal of Pain 9 (11) (November): 999-1005. doi:10.1016/ j.jpain.2008.05.010.

\section{Published Version}

doi:10.1016/j.jpain.2008.05.010

\section{Permanent link}

http://nrs.harvard.edu/urn-3:HUL.InstRepos:37058121

\section{Terms of Use}

This article was downloaded from Harvard University's DASH repository, and is made available under the terms and conditions applicable to Other Posted Material, as set forth at http:// nrs.harvard.edu/urn-3:HUL.InstRepos:dash.current.terms-of-use\#LAA

\section{Share Your Story}

The Harvard community has made this article openly available.

Please share how this access benefits you. Submit a story.

Accessibility 


\title{
Dermatomal Scratching After Intramedullary Quisqualate Injection: Correlation With Cutaneous Denervation
}

\author{
Kori L. Brewer ${ }^{\star}$, Jeung Woon Lee ${ }^{\dagger}$, Heather Downs ${ }^{\dagger}$, Anne Louise Oaklander ${ }^{\star}, \ddagger$, and Robert \\ P. Yezierski§ \\ * Department of Emergency Medicine, Brody School of Medicine at East Carolina University, \\ Greenville, North Carolina \\ † Departments of Neurology and Pathology, Massachusetts General Hospital, Boston, \\ Massachusetts \\ ‡ Department of Anesthesia and Neurology, Harvard Medical School, Boston, Massachusetts \\ $\S$ Departments of Orthodontics and Neuroscience, Comprehensive Center for Pain Research, \\ University of Florida, Gainesville, Florida
}

\begin{abstract}
Central nervous system lesions cause peripheral dysfunctions currently attributed to central cell death that compromises function of intact peripheral nerves. Injecting quisqualate (QUIS) into the rat spinal cord models spinal cord injury (SCI) and causes at-level scratching and self-injury. Such overgrooming was interpreted to model pain until patients with self-injurious scratching after SCI reported itch motivated scratching that was painless because of sensory loss. Because selfinjurious scratching is difficult to explain by central mechanisms alone, we hypothesized that QUIS injections damage peripheral axons of at-level afferents. QUIS was injected into thoracic spinal cords of 18 Long-Evans rats. Animals were killed 3 days after overgrooming began or 14 days after injection. Spinal cord lesions were localized and DRG-immunolabeled for ATF-3. Atlevel and control skin samples were PGP9.5-immunabeled to quantify axons. Eighty-four percent of QUIS rats overgroomed. Skin in these regions had lost two-thirds of epidermal innervation as compared with controls $(P<.001)$. Rats that overgroomed had $47 \%$ less axon-length than nongrooming rats $(P=.006)$. The presence of ATF-3 immunolabeled neurons within diagnosisrelated groups of QUIS rats indicated death of afferent cell bodies. Overgrooming after QUIS injections may not be due entirely to central changes. As in humans, self-injurious neuropathic scratching appeared to require loss of protective pain sensations in addition to peripheral denervation.
\end{abstract}

Perspective-This study suggests that intramedullary injection of quisqualic acid in rats causes death of at-level peripheral as well as central neurons. Self-injurious dermatomal scratching that develops in spinal-injured rats may reflect neuropathic itch and loss of protective pain sensations.

\section{Keywords}

Itch; pruritus; neuropathic pain; spinal cord injury; allodynia; ATF-3

\footnotetext{
(C) 2008 by the American Pain Society

Address reprint requests to Dr. Kori L. Brewer, Brody School of Medicine at East Carolina University, Department of Emergency Medicine, Division of Research, PCMH 3ED, 600 Moye Boulevard, Greenville, NC 27834. brewerk@ecu.edu.

Drs. Oaklander and Yezierski contributed equally to this work.

Presented in abstract form at the 4th International Workshop for the Study of Itch.
} 
Traumatic and/or ischemic spinal cord injury (SCI) causes local cell death and dysfunction from the primary insult and from initiation of excitotoxic and inflammatory cascades. ${ }^{4,10,24}$ SCI often causes sensory, autonomic, and motor dysfunction and loss, including at-level and below-level pain. $., 38,40$ Historically, SCI research has focused on the injury epicenter and adjacent tissue. Recent efforts to understand the full impact of SCI have led to studies focusing on remote sites, including cortical and subcortical regions of the brain. $1,5,19$ Potential effects of SCI on peripheral sensory function and tissues are not as wellcharacterized. Because primary afferents extend 1 of their 2 axons into the spinal cord, we investigated whether dorsal horn injury might have peripheral consequences that contribute to functional/behavioral sensory changes.

A secondary goal of this study was to further investigate the anatomical substrate for dermatomal scratching after SCI. Intramedullary injection of quisqualate (QUIS) results in spontaneous biting and scratching behavior at the dermatome associated with the level of injury. This "overgrooming" or "excessive grooming" is associated with loss of neurons in the deep dorsal horn while sparing neurons in the superficial lamina. ${ }^{36}$ Elimination of neurons in this region that express NK-1 receptors prevents or eliminates excessive grooming behavior. ${ }^{39}$ Although traditionally interpreted as a manifestation of spontaneous pain, ${ }^{36}$ a recent alternative hypothesis is that overgrooming may reflect uncontrolled scratching of neuropathic itch. ${ }^{9}$ The rationale for this proposal is that patients with neuropathic pain usually shield their affected area from contact, which can be painful (allodynia), whereas patients with neuropathic scratching report that their motivation is itch, not pain. ${ }^{22}$ Patients whose neuropathic itch has led to self-injury from their scratching (like QUIS-injected rats), report that loss of pain sensation from their injury removes the natural protection that limits scratching. ${ }^{21}$ A human correlate of overgrooming in QUIS-injected rats-dermatomal itching and scratching - has been proposed in patients with SCI from intramedullary cavernous hemangiomas. ${ }^{9,33,41}$ This has been suggested to represent central itch, since these hemangiomas were intramedullary. Hemosiderin and gliosis were posited to trigger ectopic activity of second-order itch projection neurons in dorsal horn lamina I. ${ }^{2}$

The current study examines an alternative hypothesis that QUIS injection may cause damage to at-level peripheral nerves. We evaluated whether there were peripheral axonal correlates of QUIS overgrooming using computerized stereological methods to estimate total length of PGP9.5-IR axons within the epidermis. We compared skin sampled from unoperated rats and rats that did or did not overgroom after QUIS injections. More than $90 \%$ of such epidermal neurites are TRPV1-IR nociceptors. ${ }^{29}$ Additionally, the presence of activating transcription factor-3 (ATF-3) immunoreactivity in at-level dorsal root ganglia neuronal cell bodies was assessed to determine if cutaneous neurite loss after QUIS injection represents central-distal peripheral axonopathy ${ }^{31}$ or death of entire primary afferent fibers. The results suggest that intramedullary QUIS injection, a model of SCI, causes profound peripheral nervous system (PNS) as well as central nervous system (CNS) injury, and that development of post-QUIS dermatomal overgrooming, currently used as a marker of central pain, may in addition represent neuropathic itch in profoundly denervated skin.

\section{Methods}

Experimental procedures were approved by the Institutional Animal Care and Use Committee of East Carolina University. Intramedullary injection methods were as previously described. ${ }^{36}$ Male Long-Evans rats (250-275 g) were anesthetized with isoflurane (1.5\%-2\%), shaved, scrubbed with Betadine, and wiped with $70 \%$ alcohol. A midline incision was made and muscle layers bluntly dissected to expose the junction of thoracic and lumbar spine. The spinous process and vertebral laminae were removed from the L1 and the dura incised longitudinally and reflected. Unilateral injections were made 
using glass micropipettes (tip diameter, 5-10 $\mu \mathrm{m}$ ) attached to a Hamilton syringe (volume, $10 \mu \mathrm{L}$ ). The syringe was mounted on a microinjector (Kopf 5000; Kopf, Tujunga, CA) attached to a micromanipulator. Injections were made between the dorsal vein and dorsal root entry zone at a depth of $1000 \mu \mathrm{m}$ below the spinal cord surface. A stock solution of 125 mM QUIS (Sigma, St. Louis, MO) was made using phosphate buffered saline (PBS). Each animal was injected with $1.2 \mu \mathrm{L}$ of QUIS $(\mathrm{n}=18)$, over a 60 -second interval ( 3 tracks of 0.4 $\mu \mathrm{L}$ separated by $0.3 \mathrm{~mm}$ parallel to the long axis of the cord). After injections, muscles were sutured, the skin closed with wound clips, and animals returned to their home cages.

Animals were monitored daily after surgery for the presence of self-directed biting and scratching resulting in injury to the skin and deeper tissues. ${ }^{36}$ Rats that began to overgroom were euthanized 3 days after initiation of the behavior. All other animals were killed 14 days after surgery. Rats were anesthetized by isoflurane at which time 2-mm skin punch biopsies (Acuderm, Ft. Lauderdale, FL) were removed from (1) intact skin targeted for overgrooming (including areas of skin that had minimal hair removal but without ulceration); (2) $15 \mathrm{~mm}$ proximal to the epicenter of overgrooming (an area just outside the area of grooming, ie, skin showed no signs of overgrooming); (3) the shoulder ipsilateral to overgrooming (an ipsilateral control site); and (4) the "mirror site" on the contralateral flank corresponding to the grooming area. In nonovergrooming rats, biopsy specimens were collected from the center of the dermatome corresponding to the spinal level of injection, $15 \mathrm{~mm}$ proximal to this point, and the ipsilateral shoulder. Biopsy specimens were also obtained from the same location on unoperated rats. Rats were perfused through the heart with cold saline followed by $4 \%$ paraformaldehyde. Spinal cords were removed and placed in $4 \%$ paraformaldehyde for 24 hours followed by $30 \%$ sucrose for at least 48 hours. Using a freezing microtome (Leica 2400; Leica Microsystems, Wetzler, Germany), 75- $\mu \mathrm{m}$ sections were cut through a $0.5-\mathrm{cm}$ length of the spinal cord surrounding the lesion site, collected in PBS, mounted on gelatin-coated slides, and stained with cresyl violet. Light microscopy was used to qualitatively describe damage to the spinal cord as unilateral or bilateral. Each skin sample was classified as ipsilateral or contralateral to the side of injury. Samples were further categorized as having come from a grooming or nongrooming area.

Dorsal root ganglia were also collected bilaterally from all QUIS injected rats at the level of injection and post-fixed as described above. Twenty-four diagnosis-related groups (DRGs) were randomly selected for ATF-3 staining and were serially cut into $30-\mu \mathrm{m}$ sections using a freezing microtome (Microm, Waldorf, Germany) and collected in phosphate buffer (PB). From each DRG, 3 sequential sections were processed for ATF-3 immunohistochemistry. Sections were incubated in $1 \% \mathrm{H}_{2} \mathrm{O}_{2}$, washed 3 times in $\mathrm{PB}$, and placed in blocking solution (5\% normal serum, $0.4 \%$ TX-100/PB) for 2 to 3 hours. Sections were incubated overnight with ATF-3 primary antibody (1:500; Santa Cruz Biotech, Santa Cruz, CA) diluted in blocking solution. Sections were washed 3 times in PBS, incubated for 2 hours in secondary antibody solution (Vector Labs, Burlingame, CA), then for 1.5 hours in Vectastain ABC solution (Vector Labs). DRG sections were visualized using 3'27 3'-diamino benzidine (DAB; Vector Labs), mounted on gel-coated slides, dehydrated, cleared in xylene, and coverslipped with Permount. Three $30-\mu \mathrm{m}$ serial sections were used for stereological quantification of ATF3-immunoreactive cells from each DRG (StereoInvestigator software, version 6.0; MicroBrightField Inc., Williston, VT). A $175 \times 175-\mu \mathrm{m}$ counting frame, a 175 $\times 175-\mu \mathrm{m}$ sampling grid, and an optical dissector height of $25 \mu \mathrm{m}$ was used. Cells were counted at a final magnification of $\times 600$. No DRGs were collected from unoperated rats, as there is convincing support in the literature that these tissues should not contain any ATF+ cells. ${ }^{28,32}$

Skin punches were fixed in 2\% PLP for 12 to 24 hours, rinsed in $0.1 \mathrm{M}$ Sorenson phosphate buffer, transferred to a cryoprotectant solution ( $20 \%$ glycerol, $20 \%$ 0.4M Sorenson's buffer 
and $60 \% \mathrm{dH} 20$ ), and stored at $4^{\circ} \mathrm{C}$. Fifty-micron sections were immunolabeled against PGP9.5 (Chemicon, Temecula, CA), using standard clinical methods. ${ }^{15}$ Axonal localization of epidermal PGP9.5 immunolabeling has been verified ultrastructurally ${ }^{12}$ and more than 90\% of PGP9.5-immunoreactive epidermal neurites are TRPV1 ${ }^{+}$nociceptive small-fiber axonal endings. ${ }^{29}$ Quantitative data were obtained from the epidermis where axons individuate. Slides containing $350-\mu \mathrm{m}$ transverse serial skin sections (random start; $200-\mu \mathrm{m}$ interval) were used to digitally trace the total length of PGP9. $5{ }^{+}$fibers present within the epidermal layer of all skin punches (Neurolucida software, version 6.0; MicroBrightField Inc., Williston, VT). Intraepidermal neurites branch in the rat plantar paw, so individual axons can not be counted. Hence, the total length of all intraepidermal nerve fibers including all primary and secondary branches was measured by digitally tracing them using $x-y-z$ coordinates at a final magnification of $\times 600$. The reference space was calculated from the length and thickness of each skin punch and the data expressed as total axonal length (mm) per $\mathrm{mm}^{2}$ skin surface area. Epidermal thickness was not factored in because the epidermis thins if it is denervated giving the potential for false-normal readings. ${ }^{7}$

Although all overgrooming animals had damage to the ipsilateral spinal cord, not all cord damage resulted in overgrooming. Therefore, comparisons were also made between samples corresponding to injured versus noninjured sides of the cord. Summaries of data from groups are reported as mean \pm SEM. $P<.05$ represented statistical significance. The number of positively stained neurites was compared across groups by ANOVA followed by the Fisher's PLSD post hoc analysis. The proportions of DRGs with positive ATF-3 staining were compared across groups by using a Fisher's exact test. Data was analyzed using StatView (v.5.0.1; SAS, Inc., Cary, NC).

\section{Results}

Intramedullary injection of QUIS caused neuronal loss and inflammation within the gray matter as previously described (Fig 1A). Eleven of the QUIS-injected rats had bilateral spinal cord damage, 6 had unilateral damage, and one had no evidence of an injection and was excluded from study. Of the 17 animals included, 12 developed unilateral overgrooming, 2 developed bilateral overgrooming, and 3 did not develop overgrooming. The average time to onset of overgrooming was 8 days. An example of a region of skin targeted for overgrooming behavior is shown in Fig 1B.

Eleven skin punches were obtained from unoperated control rats and data from these punches were used as control values. A total of 34 skin punches were collected from the level of injury of QUIS-injected animals (skin targeted for grooming and its mirror site, or skin from both sides at the level of injury in nongrooming animals). Of these, 27 were processed and included in the analysis. The remaining samples were not able to be analyzed due to insufficient fixation during tissue processing. Of the 27 usable punches, 13 came from regions of overgrooming. Fourteen skin samples came from ungroomed skin associated with an injected spinal segment. In 6 of 14 samples from ungroomed sites, there were no visible pathological abnormalities in the cord segment corresponding to the sampled dermatome.

Skin samples from unoperated rats had a mean total neurite length of $3241.4 \pm 342.9 \mu \mathrm{m} /$ $\mu \mathrm{m}^{2}$. Analysis of the axonal length of PGP9.5-IR labeled intraepidermal neurites revealed that QUIS-injected rats had no difference in neurite length in skin between injured and uninjured sides of the cord $\left(1403.2 \pm 173.3\right.$ vs $\left.2081.3 \pm 345.7 \mu \mathrm{m} / \mu \mathrm{m}^{2} ; P=.141\right)$. However, skin from both of these sites (corresponding to injured and uninjured sides of injected cords) had decreased neurite lengths as compared with uninjected rats $(P<.001$ and $P=.023$, respectively, Figs 2 and 3). No difference in total axonal length was observed between 
samples taken from unoperated animals and control samples from the ipsilateral shoulder of injected animals $(P=.547)$.

While neurite length in both overgroomed and ungroomed skin was reduced in QUISinjected rats as compared with unoperated controls $(1082.0 \pm 165.3$ and $2033.7 \pm 208.2$ vs $3241.4 \pm 342.9 \mu \mathrm{m} / \mu \mathrm{m}^{2} ; P<.0001$ and $P=.023$, respectively), skin from overgroomed areas had decreased neurite lengths compared with ungroomed skin $(P=.007$; Fig 3$)$. Skin from overgroomed sites ipsilateral to cord damage was less innervated than skin from sites associated with cord damage but no overgrooming $(1082.0 \pm 165.3$ vs $1999.8 \pm 280.8 \mu \mathrm{m} /$ $\left.\mu \mathrm{m}^{2} ; P=.007\right)$. Total axonal length within the epidermis of skin samples taken $15 \mathrm{~mm}$ proximal to the epicenter of overgrooming sites $(\mathrm{n}=10)$ had intermediately reduced values $\left(1887 \pm 228.36 \mu \mathrm{m} / \mu \mathrm{m}^{2} ; P=.012\right)$, similar to those in samples from ungroomed skin from injected animals.

Of the 34 DRGs collected from QUIS-injected animals, ATF-3 staining was performed on 12 taken from the side of cords corresponding to an area targeted for overgrooming. Twelve came from sides of cords with no overgrooming. ATF- 3 immunoreactive neuronal profiles were present in 17/24 DRGs studied (70.8\%). Fourteen of 17 positively-labeled DRGs came from the damaged side of the spinal cord, and 3 of 17 from an uninjured side of cord. Eighty-three percent of DRGs taken from a side targeted for overgrooming $(\mathrm{n}=10)$ had immunolabeling compared with 58.3\% from ungroomed sides ( $\mathrm{n}=7$; Fisher's exact, $P=$. 202). The mean estimated value of the number of ATF-3 immunoreactive cells for DRGs collected from sides associated with overgrooming was $17.2 \pm 9.0$ vs $11.4 \pm 4.5$ ATF- $3+$ DRGs from ungroomed sides $(P=.36)$. There was no correlation between the presence of ATF-3 immunoreactivity and peripheral neurite density $(P=.344)$ or overgrooming behavior $(P=.192)$.

\section{Discussion}

These results demonstrate that intramedullary QUIS injection causes loss of markers for peripheral axons both ipsilaterally and contralaterally to QUIS injections. The vast majority (83\%) of DRGs collected from spinal cord segments damaged by QUIS injection contained neuronal profiles labeled for ATF-3, suggesting that distal axonal loss measured in the skin was a manifestation of death of the entire primary afferent neuron (a neuronopathy rather than an axonopathy). Although our data did not show a significant correlation between positive ATF-3 staining and peripheral PGP9.5 staining or overgrooming behavior, this may be the result of long postinjury survival times. Most studies indicate that ATF-3 labeling peaks within 4 days of injury and diminishes rapidly by 8 to 10 days. Average survival time for rats in the present study was 14.8 days (range, 8-23 days), making it likely that the bulk of the cell death had occurred before animal euthanizing and immunohistological processing. Because there was still ATF-3 staining present under these suboptimal conditions, we believe the QUIS model of SCI effectively creates a central-to-peripheral pathological injury with behavioral concomitants. The current results provide evidence for peripheral consequences of CNS lesions and provide a potential additional mechanism for at-level somatosensory dysfunction associated with spinal cord injury. There is preliminary evidence that even more rostral lesions can cause trans-synaptic retrograde degeneration of peripheral cutaneous afferents. ${ }^{6,34}$ The mechanisms by which this might happen are unknown.

An unexpected finding was that unilateral QUIS injections also affected the epidermal axons on the side contralateral to the cord damage. This suggests a secondary process independent of neuronal and axonal degeneration within the dorsal horn. Our method of spinal cord histological analysis does not detect subcellular changes in neuronal viability or function, 
nor changes in gene or protein expression, so it is not possible to know if the "uninjured" side of the spinal cord was fully normal or might have early degenerative changes. This and previous studies have shown that unilateral QUIS injections often cause bilateral spinal cord pathology that develops slowly (Fig 1A). Indirect support for contralateral cord injury comes from observations that rats with pathologically unilateral cord injuries can have bilateral alterations in mechanical and thermal thresholds in regions caudal to the injury. ${ }^{36}$ Therefore, it is possible that an early, sublethal effect of the QUIS-induced injury cascade triggers loss of primary afferents. This is supported by our observation that $18 \%$ of ATF-3 positive DRG came from sides of the cord that lacked visible pathology. Alternatively, many bilateral effects of unilateral distal peripheral lesions that do not involve direct spinal cord injury have been described in many species, including humans. ${ }^{14,23}$

An important observation of this study was the association between loss of cutaneous axons and the development of self-injurious overgrooming. Overgrooming behavior arose in the context of near-total loss of peripheral innervation. This association has also been found in some patients who self-injure by scratching skin afflicted by neuropathic itch after peripheral nerve injuries such as shingles..$^{21}$ It should be noted that the area of skin targeted for overgrooming is not insensate, as mechanical stimulation of this region produces responses in dorsal horn neurons ${ }^{37}$ and overgrooming behavior can be attenuated and even reversed as previously described ${ }^{39}$ Therefore, this behavior is unlikely to represent a condition similar to autotomy. The fact that overgrooming and denervation are restricted to the dermatome corresponding to the level of injury suggests that these conditions appear to be an "at-level" rather than "below-level" consequence of spinal cord injury, consistent with the idea that some remaining afferent drive, or edge effects at transitions between normal and denervated skin may produce neuropathic itch. In the present study, cutaneous denervation was most severe in skin targeted for overgrooming, suggesting the possibility of a "denervation threshold" for the initiation of overgrooming. Previous studies related to the QUIS model have identified a "spatial threshold" of spinal cord damage needed in order to elicit overgrooming. ${ }^{36}$ In this study, no animal with an average neurite length of over 2300 $\mu \mathrm{m} / \mu \mathrm{m}^{2}$ developed overgrooming. Based on the current data, the concept of threshold effects may extend to include peripheral neurite loss in addition to a central component involving the longitudinal spread of pathology.

Recently, overgrooming in the QUIS model of SCI was suggested to model scratching from central neuropathic itch. ${ }^{9}$ In addition to dermatological and medical conditions, chronic itch can be caused by disorders of the CNS or PNS. ${ }^{3,21,35,42}$ A subset of patients with neuropathic itch develop scratching that persists despite self injury. ${ }^{16}$ Self-injurious behavior in humans is typically restricted to individuals with severe denervation, consistent with a loss of protective pain sensations. ${ }^{17,26}$ Recently, Symons et al ${ }^{30}$ reported selfinjurious behavior in patients with neurodevelopmental disorders to be correlated with "morphologic abnormalities" in epidermal nerve fibers, including altered distribution of fibers in the skin of patients with this behavior. Therefore, QUIS injections may be modeling a condition of severe pathological itch, with its strong drive to scratch, coupled with loss of protective pain fibers, leading to self-injury. Consistent with this conclusion is the observation that loss of sensory PNS axons can result in hallucinations specific to the modality that is lost. ${ }^{18,27}$ Notably, postherpetic neuralgia is reported to develop in the subset of shingles patients with the most severe loss of nociceptive axons in skin biopsies. ${ }^{11,20,25}$

In the case of QUIS injections, overgrooming most reliably occurs when damage to the cord is restricted to the deeper layers of the dorsal horn, sparing the superficial laminae. ${ }^{36}$ This type of lesion has been shown to generate abnormal activity in lamina I cells and destruction of these cells can prevent or eliminate overgrooming behavior. ${ }^{39}$ Recent evidence has demonstrated the existence of a pathway that is anatomically congruent to known pain 
pathways but responds to itch through input from histamine-sensitive C-fibers. ${ }^{2}$ Therefore, the overgrooming response to QUIS injection may result from injury induced spontaneous activity in second order "itch neurons" in lamina I of the dorsal horn" that are responding to not only the loss of central input but also loss of normal peripheral input. It should be noted that pain is much more common after SCI than itch, ${ }^{38,40}$ and the population of spinothalamic projection neurons that subserve itch is relatively small. ${ }^{2,13}$ Pain can be present in QUIS-injected animals without overgrooming, as evidenced by the finding that many injected animals become hypersensitive to thermal and mechanical stimuli applied below the level of injury without any signs of overgrooming at the level of injury. On the other hand, overgrooming animals are found to consistently have allodynia and hyperalgesia. ${ }^{36}$

In conclusion, intramedullary injections of QUIS leads to degeneration of peripheral sensory axons in the dermatome associated with injury, presumably initiated by injury to the terminals of central axons. ATF-3 immunolabeling in DRG suggests that this involves at least in part, degeneration of neuronal cell bodies. Dermatomal overgrooming is associated with the most severe loss of cutaneous innervation. These results support the use of overgrooming behavior after excitotoxic SCI as a model to study chronic aversive sensations after spinal injury. In the future it will be important to evaluate whether overgrooming is primarily associated with degeneration of peripheral unmyelinated axons as well as identify specific central changes responsible for this behavior.

\section{Acknowledgments}

Supported by the Public Health Service (R01NS42866, K24NS059892, P30 EY 12196) and University of Florida Comprehensive Center for Pain Research.

\section{References}

1. Abraham KE, McGinty JF, Brewer KL. Opioid peptide mRNA expression is related to the onset of pain-related behaviors following excitotoxic spinal cord injury. Pain. 2001; 90:181-190. [PubMed: 11166985]

2. Andrew D, Craig AD. Spinothalamic lamina I neurons selectively sensitive to histamine: A central neural pathway for itch. Nat Neurosci. 2001; 4:72-77. [PubMed: 11135647]

3. Bernhard JD. Itch and pruritus: What are they and how should itches be classified? Dermatol Ther. 2005; 18:288-291. [PubMed: 16296999]

4. Blight AR. Morphometric analysis of a model of spinal cord injury in guinea pigs, with behavioral evidence of delayed secondary pathology. J Neurol Sci. 1991; 103:156-71. [PubMed: 1880533]

5. Brewer KL, Nolan T. Spinal and supraspinal expression of tumor necrosis factor-alpha (TNF- $\alpha$ ) following excitotoxic spinal cord injury. J Mol Neurosci. 2007; 31:13-22. [PubMed: 17416966]

6. Chémali KR, Zhou L. Small fiber degeneration in post-stroke complex regional pain syndrome I. Neurology. 2007; 69:316-317. [PubMed: 17636073]

7. Chiang HY, Huang IT, Chen WP, Chien HF, Shun CT, Chang YC, Hsieh ST. Regional difference in epidermal thinning after skin denervation. Exp Neurol. 1998; 154:137-145. [PubMed: 9875275]

8. Christensen MD, Hulsebosch CE. Chronic pain after spinal cord injury. J Neurotrauma. 1997; 14:517-537. [PubMed: 9300563]

9. Dey DD, Landrum O, Oaklander AL. Central neuropathic itch from spinal cord cavernous hemangioma: A human case, a possible animal model, and a hypotheses about pathogenesis. Pain. 2005; 113:233-237. [PubMed: 15621384]

10. Dusart I, Schwab ME. Secondary cell death and the inflammatory reaction after dorsal hemisection of the rat spinal cord. Eur J Neurosci. 1994; 6:712-724. [PubMed: 8075816]

11. Fields HL, Rowbotham M, Baron R. Postherpetic neuralgia: Irritable nociceptors and deafferentation. Neurobiol Dis. 1998; 5:205-214. 
12. Hilliges M, Wang L, Johansson O. Ultrastructural evidence for nerve fibers within all vital layers of the human epidermis. J Invest Dermatol. 1995; 104:134-137. [PubMed: 7798631]

13. Ikoma A, Steinhoff M, Stander S, Yosipovitch G, Schmelz M. The neurobiology of itch. Nat Rev Neurosci. 2006; 7:535-547. [PubMed: 16791143]

14. Koltzenburg M, Wall PD, McMahon SB. Does the right side know what the left is doing? Trends Neurosci. 1999; 22:122-127. [PubMed: 10199637]

15. Lauria G, Lombardi R, Borgna M, Penza P, Bianchi R, Savino C, Canta A, Nicolini G, Marmiroli $P$, Cavaletti G. Intraepidermal nerve fiber density in rat foot pad: Neuropathologicneurophysiologic correlation. J Peripher Nerv Syst. 2005; 10:202-208. [PubMed: 15958131]

16. Mailis A. Compulsive targeted self-injurious behavior in humans with neuropathic pain: A counterpart of animal autotomy? Four case reports and literature review. Pain. 1996; 64:569-578. [PubMed: 8783323]

17. McCarthy BG, Hsieh ST, Stocks A, Hauer P, Macko C, Cornblath DR, Griffin JW, McArthur JC. Cutaneous innervation in sensory neuropathies: Evaluation by skin biopsies. Neurology. 1995; 45:1848-1855. [PubMed: 7477980]

18. Møller AR. Similarities between pain and tinnitus. Am J Otol. 1997; 18:268-289. [PubMed: 9093691]

19. Morrow TJ, Paulson PE, Brewer KL, Yezierski RP, Casey KL. Chronic, selective forebrain responses to excitotoxic dorsal horn injury. Exp Neurol. 2000; 161:220-226. [PubMed: 10683288]

20. Oaklander AL, Romans K, Horasek S, Stocks A, Hauer P, Meyer RA. Unilateral postherpetic neuralgia is associated with bilateral sensory neuron damage. Ann Neurol. 1998; 44:789-795. [PubMed: 9818935]

21. Oaklander AL, Cohen SP, Raju SV. Intractable postherpetic itch and cutaneous deafferentation after facial shingles. Pain. 2002; 96:9-12. [PubMed: 11932056]

22. Oaklander AL, Bowsher D, Galer B, Haanpää M, Jensen MP. Herpes zoster itch: Preliminary epidemiologic data. J Pain. 2003; 4:338-343. [PubMed: 14622691]

23. Oaklander AL, Brown JM. Unilateral nerve injury produces bilateral loss of distal innervation. Ann Neurol. 2004; 55:639-644. [PubMed: 15122703]

24. Popovich PG, Wei P, Stokes BT. Cellular inflammatory response after spinal cord injury in Sprague-Dawley and Lewis rats. J Comp Neurol. 1997; 377:443-464. [PubMed: 8989657]

25. Rowbotham MC, Yosipovitch G, Connolly MK, Finlay D, Forde G, Fields HL. Cutaneous innervation density in the allodynic form of postherpetic neuralgia. Neurobiol Dis. 1996; 3:205214. [PubMed: 8980021]

26. Schmahl C, Greffrath W, Baumgartner U, Schlereth T, Magerl W, Philipsen A, Lieb K, Bohus M, Treed RD. Differential nociceptive deficits in patients with borderline personality disorders and self-injurious behavior: Laser-evoked potentials, patient discrimination of noxious stimuli, and pain ratings. Pain. 2004; 110:470-479. [PubMed: 15275800]

27. Schultz G, Melzack R. Visual hallucinations and mental state: A study of 14 Charles Bonnet syndrome hallucinators. J Nerv Ment Dis. 1993; 181:639-643. [PubMed: 8409963]

28. Seijffers R, Allchorne AJ, Woolf CJ. The transcription factor ATF-3 promotes neurite outgrowth. Mol Cell Neurosci. 2006; 32:143-154. [PubMed: 16713293]

29. Simone DA, Nolano M, Johnson T, Wendelschafer-Crabb G, Kennedy WR. Intradermal injection of capsaicin in humans produces degeneration and subsequent reinnervation of epidermal nerve fibers: Correlation with sensory function. J Neurosci. 2005; 18:8947-8959. [PubMed: 9787000]

30. Symons FJ, Wendelschafer-Crabb G, Kennedy W, Hardrict R, Dahl N, Bodfish JW. Evidence of altered epidermal nerve fiber morphology in adults with self-injurious behavior and neurodevelopmental disorders. Pain. 2008; 134:232-237. [PubMed: 17850969]

31. Thomas PK. Selective vulnerability of the centrifugal and centripetal axons of primary sensory neurons. Muscle Nerve. 1982; 5:S117-S121. [PubMed: 6221191]

32. Tsujino H, Kondo E, Fukuoka T, Dai Y, Tokunaga A, Miki K, Yonenobu K, Ochi T, Noguchi K. Activating transcription factor 3 (ATF3) induction by axotomy in sensory and motoneurons: A novel neuronal marker of nerve injury. Mol Cell Neurosci. 2000; 15:170-182. [PubMed: 10673325] 
33. Vuadens P, Regli F, Dolivo M, Uske A. Segmental pruritus and intramedullary vascular malformation. Schweiz Arch Neruol Psychiatr. 1994; 145:13-16.

34. Wallengren J, Tegner E, Sundler F. Cutaneous sensory nerve fibers are decreased in number after peripheral and central nerve damage. J Am Acad Dermatol. 2002; 46:215-217. [PubMed: 11807432]

35. Yamamoto M, Yabuki S, Hayabara T, Otsuki S. Paroxysmal itching in multiple sclerosis: A report of three cases. J Neurol Neurosurg Psychiatry. 1981; 44:19-22. [PubMed: 7205301]

36. Yezierski RP, Liu S, Ruenes GL, Kajander KJ, Brewer KL. Excitotoxic spinal cord injury: Behavioral and morphological characteristics of a central pain model. Pain. 1998; 75:141-155. [PubMed: 9539683]

37. Yezierski RP, Park SH. The mechanosensitivity of spinal sensory neurons following intramedullary injections of quisqualic acid in the rat. Neurosci Lett. 1993; 157:115-119. [PubMed: 8233021]

38. Yezierski RP. Pain following spinal cord injury: Pathophysiology and central mechanisms. Prog Brain Res. 2000; 129:429-449. [PubMed: 11098709]

39. Yezierski RP, Yu C-G, Manthy PW, Vierck C, Lappi DA. Spinal neurons involved in the generation of at-level pain following spinal injury in the rat. Neurosci Lett. 2004; 361:232-236. [PubMed: 15135936]

40. Yezierski RP. Spinal cord injury: A model of central neuropathic pain. Neurosignals. 2005; 14:182-193. [PubMed: 16215301]

41. Yezierski RP, Vierck C. Comment on: Central neuropathic itch from spinal cord cavernous hemangioma: A human case, a possible animal model, and hypotheses about pathogenesis. Pain. 2005; 155:419-421. [PubMed: 15878800]

42. Yosipovitch G, Fleischer AB. Itch associated with skin disease: Advances in pathophysiology and emerging therapies. Am J Clin Dermatol. 2003; 4:617-622. [PubMed: 12926980] 

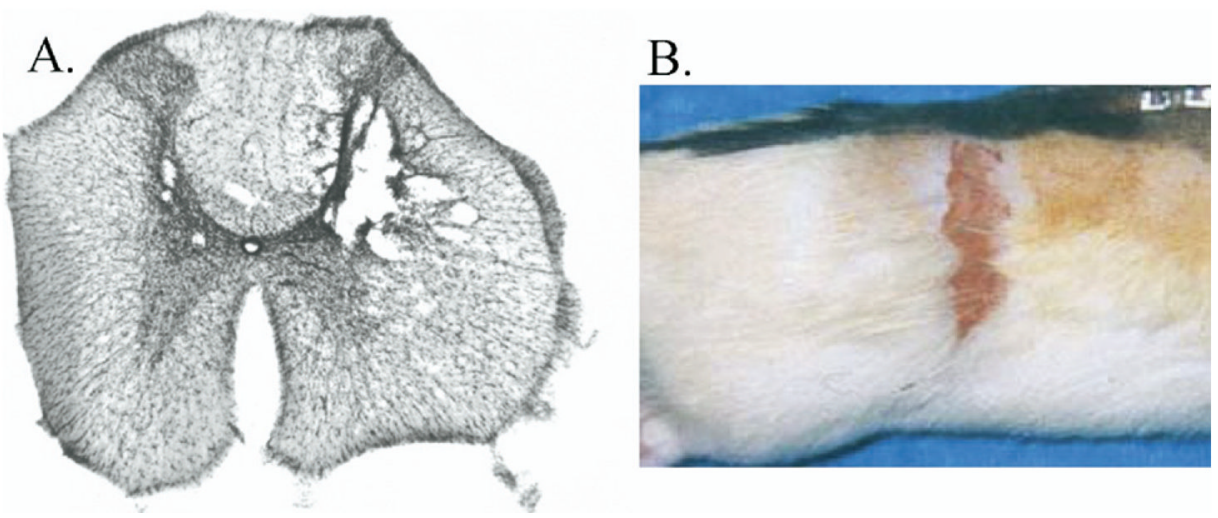

Figure 1.

A, Cresyl violet staining of spinal cord 9 days after intramedullary injection of quisqualate (QUIS) into the right dorsal gray matter at approximate spinal level T13. Cell loss within laminae III-V is evident on both sides of the cord. B, Photograph of a QUIS-injected rat 5 days after onset of dermatomal overgrooming. 

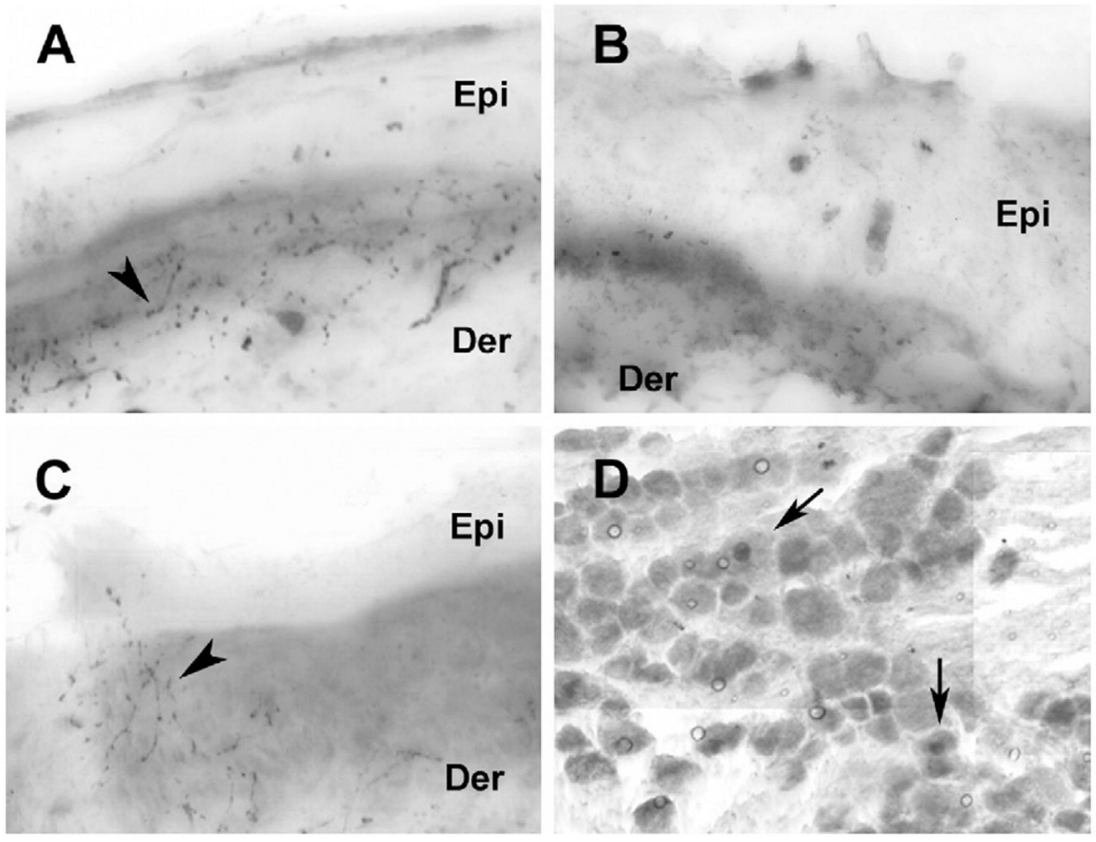

Figure 2.

Photomicrographs showing PGP95 ${ }^{+}$fibers of sampled skin tissues (A-C; arrowhead) and $\mathrm{ATF}^{+}$cells from an ipsilesional diagnosis-related group (D; arrow) 2 weeks after quisqualate (QUIS) injection. Dense PGP95-immunoreactive nerve fibers were observed in the epidermal and dermal layers from control skin (A). Nongroomed QUIS-injured skin contained fewer PGP95-immunoreactive nerve fibers (C), and groomed QUIS-lesioned skin contained almost no PGP95-immunoreactive nerve fibers (B). Presence of ATF3+ cells were observed from a diagnosis-related group ipsilesional to grooming. Epi, epidermis; Der, dermis. 


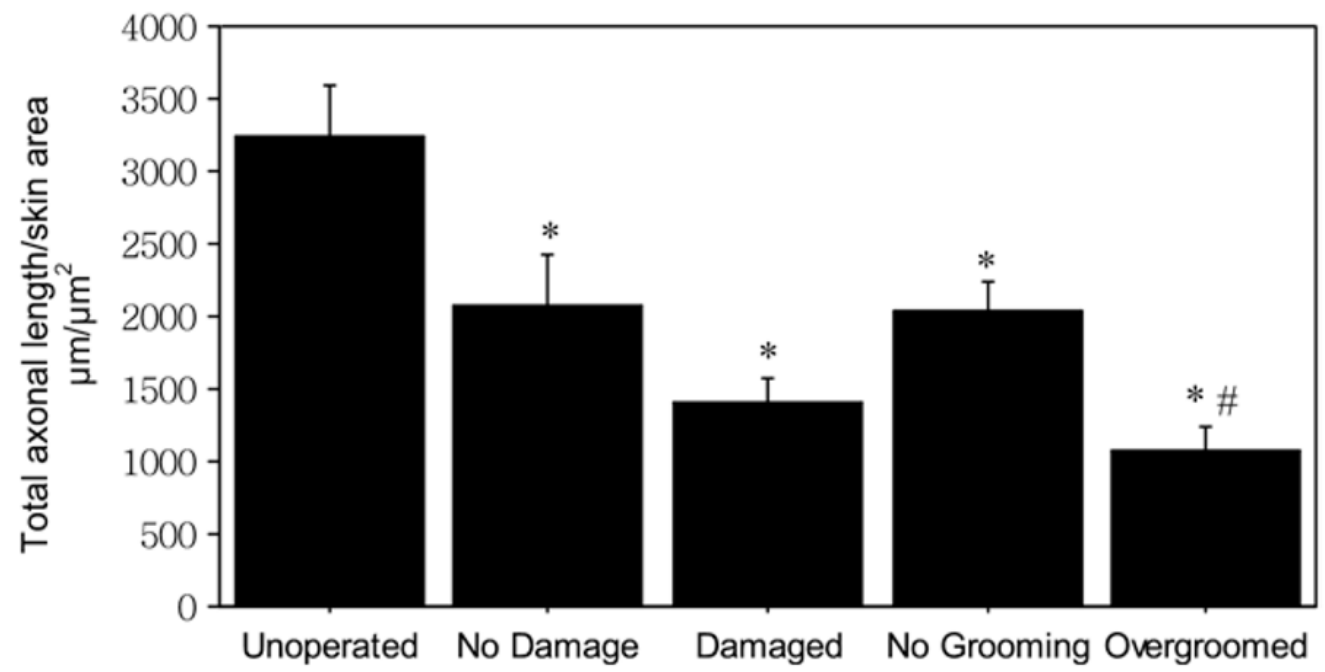

Figure 3.

Mean axonal length in skin taken from unoperated rats $(\mathrm{n}=11)$, rats injected with quisqualate (QUIS) with no signs of gross pathology on the side ipsilateral to skin biopsy (n $=5$ ), rats injected with QUIS with obvious pathology on the side ipsilateral to skin biopsy (n $=20$ ), ungroomed regions after QUIS injection $(n=7)$, and skin targeted for overgrooming after QUIS injection $(\mathrm{n}=13)$. All skin taken from QUIS-injected animals had reduced neurite density compared with unoperated controls. Skin that was overgroomed after QUIS injection had significantly reduced neurite density compared with ungroomed skin from QUIS-injected animals. *Significant difference from unoperated; ${ }^{*}$ Significant difference from ungroomed skin $(P<.01)$. 